\title{
Chiral three-nucleon forces and the evolution of correlations along the oxygen isotopic chain
}

\author{
A. Cipollone, ${ }^{1,2}$ C. Barbieri, ${ }^{1, *}$ and P. Navrátil ${ }^{3}$ \\ ${ }^{1}$ Department of Physics, University of Surrey, Guildford GU2 7XH, United Kingdom \\ ${ }^{2}$ Numerical Applications and Scenarios Division, CMCC, Bologna 40127, Italy \\ ${ }^{3}$ TRIUMF, 4004 Westbrook Mall, Vancouver, British Columbia, Canada V6T $2 A 3$
}

(Received 1 December 2014; revised manuscript received 13 May 2015; published 10 July 2015)

\begin{abstract}
Background: Three-nucleon forces ( $3 \mathrm{NFs}$ ) have nontrivial implications on the evolution of correlations at extreme proton-neutron asymmetries. Recent $a b$ initio calculations show that leading-order chiral interactions are crucial to obtain the correct binding energies and neutron driplines along the $\mathrm{O}, \mathrm{N}$, and $\mathrm{F}$ chains [A. Cipollone, C. Barbieri, and P. Navrátil, Phys. Rev. Lett. 111, 062501 (2013)].

Purpose: Here we discuss the impact of $3 \mathrm{NFs}$ along the oxygen chain for other quantities of interest, such has the spectral distribution for attachment and removal of a nucleon, spectroscopic factors, and radii. The objective is to better delineate the general effects of $3 \mathrm{NFs}$ on nuclear correlations.

Methods: We employ self-consistent Green's function (SCGF) theory which allows a comprehensive calculation of the single-particle spectral function. For the closed subshell isotopes, ${ }^{14} \mathrm{O},{ }^{16} \mathrm{O},{ }^{22} \mathrm{O},{ }^{24} \mathrm{O}$, and ${ }^{28} \mathrm{O}$, we perform calculations with the Dyson-ADC(3) method, which is fully nonperturbative and is the state of the art for both nuclear physics and quantum chemistry applications. The remaining open-shell isotopes are studied using the newly developed Gorkov-SCGF formalism up to second order.

Results: We produce complete plots for the spectral distributions. The spectroscopic factors for the dominant quasiparticle peaks are found to depend very little on the leading-order (NNLO) chiral 3NFs. The latter have small impact on the calculated matter radii, which, however, are consistently obtained smaller than experiment. Similarly, single-particle spectra tend to be too spread with respect to the experiment. This effect might hinder, to some extent, the onset of correlations and screen the quenching of calculated spectroscopic factors. The most important effect of $3 \mathrm{NFs}$ is thus the fine tuning of the energies for the dominant quasiparticle states, which governs the shell evolution and the position of driplines.

Conclusions: Although present chiral NNLO 3NFs interactions do reproduce the binding energies correctly in this mass region, the details of the nuclear spectral function remain at odds with the experiment showing too-small radii and a too-spread single-particle spectrum, similar to what has already been pointed out for larger masses. This suggests a lack of repulsion in the present model of $N N+3 N$ interactions, which is mildly apparent already for masses in the $A=14-28$ mass range.
\end{abstract}

DOI: 10.1103/PhysRevC.92.014306

PACS number(s): 21.30.Fe, 21.60.De, 05.10.Cc

\section{INTRODUCTION}

The concept of correlations is fundamental to a deep understanding of nuclear phenomena [1]. These are generally defined as characteristics of the nucleus that cannot be explained in terms of a simple mean-field picture (i.e., a wave function of Slater determinant type). These effects are often quantified in terms of the fragmentation of the single-particle strength observed when adding or removing a nucleon. An intriguing feature is the persistence of dominant quasiparticle peaks near the Fermi surface while broader resonances are found at higher excitations. This is at the origin of the duality between the liquid drop and the shell-model behavior of atomic nuclei.

Historically, several electron scattering studies have provided a wealth of information on nuclear spectral functions (see Refs. [2-9] and references therein). This has allowed a rather complete characterization of correlations for stable nuclei [1]. However, a similar full characterization for exotic isotopes is still lacking. Recent data from radioactive beam

\footnotetext{
*c.barbieri@surrey.ac.uk
}

facilities have put in evidence new phenomena such as shell evolution with changing proton-neutron asymmetry [10] and the insurgence of new magic numbers [11-13]. From the theoretical point of view, some of these effects have been explained in terms of properties of the tensor interaction [14], and the need for contributions from three-nucleon forces (3NFs) has also been pointed out [15]. More recently, it has been shown that $3 \mathrm{NFs}$ are crucial for understanding the neutron-rich side of the nuclear chart, in particular to explain the oxygen dripline at ${ }^{24} \mathrm{O}$ [16-19] and neutron-rich $\mathrm{Ca}$ isotopes [20-23]. Reference [19] found that the same mechanism responsible for the anomalous oxygen dripline also affects $\mathrm{N}$ and $\mathrm{F}$ isotopes up to at least ${ }^{29} \mathrm{~F}$, which is strongly neutron rich but still not at the dripline.

$A b$ initio calculations of atomic nuclei have advanced dramatically in the medium-mass region. Several approaches such as coupled cluster [24,25], in-medium similarity renormalization group (IM-SRG) [26], and self-consistent Green's function (SCGF) $[19,27]$ theories are now capable of approaching masses up to $A \approx 100$ or more. These make it possible to fully exploit modern chiral interactions with two-nucleon $(N N)$ forces and 3NFs evolved through SRG techniques [28]. Moreover, open-shell nuclei have become accessible through 
a Gorkov extension of the SCGF formalism [22,29,30], multireference IM-SRG [18,23], and Bogoliubov coupled cluster (BCC) [31]. These $a b$ initio studies have mainly focused on ground-state properties, such as total binding energies and $N N$ separation energies. More recent works have addressed the construction of effective shell-model interactions directly from full $N N$ plus 3 NF Hamiltonians [32,33]. This makes it possible to successfully address low-energy excitations directly from first principles.

The SCGF method has the added advantage of providing consistent optical potentials and spectral functions over the whole energy spectrum (i.e., both close and far form the Fermi surface). This gives comprehensive insights into the many-body dynamics and makes it possible to address other quantities such as giant resonances or the qualitative features of single-particle distribution [2,34], which can require considering several major shells $[27,35]$.

In this paper, we consider the Green's functions of the oxygen isotopes already obtained in Ref. [19] and extend these calculations to the remaining even-mass - and open-shellisotopes, using the Gorkov-SCGF approach. We then present first fully microscopic calculations of the evolution of the single-particle spectral functions along a complete isotopic chain. This gives an overall description of the evolution of nuclear correlations between two extremes of the nuclear chart. At the same time, it makes it possible to perform a more thorough test of modern chiral interactions and, in particular, we investigate the effects of initial 3NFs at NNLO.

For completeness, Sec. II discusses the relevant features of the SCGF formalism. It reviews the links of propagators with the spectral function and other quantities of experimental interest. Calculations are done in an $a b$ initio fashion and we discuss in some detail the choice of the Hamiltonian, the approximations taken and the expected uncertainties, when these can be estimated. This is done in Sec. III. Section IV discusses our results for single-particle spectra, spectroscopic factors, and binding energies. Full three-dimensional plots of spectral functions are discussed in the Appendix for completeness and conclusions are drawn in Sec. V.

\section{SCGF FORMALISM}

Information about the single-particle dynamics is fully contained in the one-body Green's function, or propagator, whose Lehmann representation reads

$$
\begin{aligned}
g_{\alpha \beta}(\omega)= & \sum_{n} \frac{\left\langle\Psi_{0}^{A}\left|a_{\alpha}\right| \Psi_{n}^{A+1}\right\rangle\left\langle\Psi_{n}^{A+1}\left|a_{\beta}^{\dagger}\right| \Psi_{0}^{A}\right\rangle}{\omega-\varepsilon_{n}^{+}+i \eta} \\
& +\sum_{k} \frac{\left\langle\Psi_{0}^{A}\left|a_{\beta}^{\dagger}\right| \Psi_{k}^{A-1}\right\rangle\left\langle\Psi_{k}^{A-1}\left|a_{\alpha}\right| \Psi_{0}^{A}\right\rangle}{\omega-\varepsilon_{k}^{-}-i \eta} .
\end{aligned}
$$

In Eq. (1), $\left|\Psi_{0}^{A}\right\rangle$ represents the ground state of $A$ nucleons and $\left|\Psi_{n}^{A+1}\right\rangle,\left|\Psi_{k}^{A-1}\right\rangle$ are the eigenstates of the $(A \pm 1)$-nucleon system. The greek indices $\alpha, \beta, \ldots$, label a complete orthonormal single-particle basis, while $\varepsilon_{n}^{+} \equiv\left(E_{n}^{A+1}-E_{0}^{A}\right)$ and $\varepsilon_{k}^{-} \equiv$ $\left(E_{0}^{A}-E_{k}^{A-1}\right)$ are one-nucleon addition and removal energies, respectively. Note that these are generically referred to in the literature as either "separation" or "quasiparticle" energies although the first naming normally labels transitions involving only $(A \pm 1)$-nucleon ground states. We use the second convention in the following, unless the two names are strictly equivalent. The transition amplitudes $\mathcal{X}_{\alpha}^{n} \equiv\left\langle\Psi_{n}^{A+1}\left|a_{\alpha}^{\dagger}\right| \Psi_{0}^{A}\right\rangle$ and $\mathcal{Y}_{\alpha}^{k} \equiv\left\langle\Psi_{k}^{A-1}\left|a_{\alpha}\right| \Psi_{0}^{A}\right\rangle$ give information about the strength of the corresponding particle addition and removal processes.

The one-body Green's function (1) is completely determined by solving the Dyson equation,

$$
g_{\alpha \beta}(\omega)=g_{\alpha \beta}^{0}(\omega)+\sum_{\gamma \delta} g_{\alpha \gamma}^{0}(\omega) \Sigma_{\gamma \delta}^{\star}(\omega) g_{\delta \beta}(\omega),
$$

where the unperturbed propagator $g_{\alpha \beta}^{0}(\omega)$ is the initial reference state (usually a mean-field or Hartree-Fock state), while $g_{\alpha \beta}(\omega)$ is the correlated propagator.

A full knowledge of the self-energy $\Sigma_{\alpha \beta}^{\star}(\omega)$ yields the exact solution for $g_{\alpha \beta}(\omega)$. However, in practical calculations this has to be approximated and it is expanded in terms of the propagator itself (that is, $\Sigma^{\star}=\Sigma^{\star}[g(\omega)]$ ). Thus, an iterative procedure is required to solve for $\Sigma^{\star}(\omega)$ and Eq. (2) self-consistently. The approximation schemes we employ to calculate the self-energy are outlined in the next subsection.

The attractive feature of the SCGF approach is that $g_{\alpha \beta}(\omega)$ describes the one-body dynamics completely. The particle and hole spectral functions are extracted directly from Eq. (1), respectively:

$$
\begin{aligned}
& S_{\alpha \beta}^{p}(\omega)=\sum_{n}\left(\mathcal{X}_{\alpha}^{n}\right)^{*} \mathcal{X}_{\beta}^{n} \delta\left[\omega-\left(E_{n}^{A+1}-E_{0}^{A}\right)\right], \\
& S_{\alpha \beta}^{h}(\omega)=\sum_{k} \mathcal{Y}_{\alpha}^{k}\left(\mathcal{Y}_{\beta}^{k}\right)^{*} \delta\left[\omega-\left(E_{0}^{A}-E_{k}^{A-1}\right)\right] .
\end{aligned}
$$

Any one-body observable can be calculated via the one-body density matrix $\rho_{\alpha \beta}$, which is obtained from $g_{\alpha \beta}$ as follows:

$$
\begin{aligned}
\rho_{\alpha \beta} & \equiv\left\langle\Psi_{0}^{A}\left|a_{\beta}^{\dagger} a_{\alpha}\right| \Psi_{0}^{A}\right\rangle \\
& =\int_{-\infty}^{\varepsilon_{0}^{-}} S_{\alpha \beta}^{h}(\omega) d \omega=\sum_{k}\left(\mathcal{Y}_{\beta}^{k}\right)^{*} \mathcal{Y}_{\alpha}^{k} .
\end{aligned}
$$

The expectation value of a one-body operator, $\hat{O}^{1 B}$, can then be written in terms of the $\mathcal{Y}$ amplitudes as

$$
\left\langle\hat{O}^{1 B}\right\rangle=\sum_{\alpha \beta} O_{\alpha \beta}^{1 B} \rho_{\beta \alpha}=\sum_{k} \sum_{\alpha \beta}\left(\mathcal{Y}_{\alpha}^{k}\right)^{*} O_{\alpha, \beta}^{1 B} \mathcal{Y}_{\beta}^{k} .
$$

Evaluating two- and many-nucleon observables requires the knowledge of many-body propagators. In the following, we do this by approximating the corresponding $A$-body density matrices with $A$ correlated but noninteracting propagators [Eq. (4)]. Specifically, we use this to account for the centerof-mass (c.m.) correction when calculating root mean square (rms) radii,

$$
\begin{aligned}
\left\langle r^{2}\right\rangle \simeq & \frac{1}{A} \sum_{\alpha \beta} u_{\alpha}\left\langle\alpha\left|\overrightarrow{\mathbf{r}}^{2}\right| \beta\right\rangle \rho_{\beta \alpha} \\
& -\frac{1}{A^{2}} \sum_{\alpha \beta \gamma \delta} u_{\alpha \gamma}\left\langle\alpha \gamma\left|\overrightarrow{\mathbf{r}}_{1} \cdot \overrightarrow{\mathbf{r}}_{2}\right| \beta \delta\right\rangle \rho_{\beta \alpha} \rho_{\delta \gamma},
\end{aligned}
$$

where $\overrightarrow{\mathbf{r}}_{i}$ represents the position of particle $i$. The factors $u_{\alpha}$ and $u_{\alpha \beta}$ in Eq. (6) and the two-body correction term arise 
because the intrinsic radius is calculated with respect to the c.m. of the system [36]. Point-matter radii are calculated by taking $u_{\alpha}^{\mathrm{pt}-\mathrm{m}}=(A-1) / A$ and $u_{\alpha \gamma}^{\mathrm{pt}-\mathrm{m}}=1$, while point-proton radii are found using

$$
u_{\alpha}^{\mathrm{pt}-p}= \begin{cases}\frac{A(A-2)+Z}{Z A} & \text { if } \quad \alpha \text { labels a proton state, } \\ \frac{1}{A} & \text { if } \quad \alpha \text { is a neutron, }\end{cases}
$$

and

$$
u_{\alpha \gamma}^{\mathrm{pt}-p}= \begin{cases}\frac{2 A-Z}{Z} & \text { if } \quad \alpha, \gamma \text { label two proton states, } \\ \frac{A-Z}{Z} & \text { if } \quad \alpha, \gamma \text { are a proton and a neutron, } \\ -1 & \text { if } \quad \alpha, \gamma \text { are two neutrons. }\end{cases}
$$

To obtain charge radii, we first calculate the point-proton ones and then account for the rms charge radii of the nucleons and for the Darvin-Foldy relativistic correction [37],

$$
\left\langle r_{\mathrm{ch}}^{2}\right\rangle=\left\langle r_{\mathrm{pt}-p}^{2}\right\rangle+\left\langle R_{p}^{2}\right\rangle+\frac{N}{Z}\left\langle R_{n}^{2}\right\rangle+\frac{3 \hbar^{2}}{4 m_{p}^{2} c^{2}},
$$

with $\left\langle R_{p}^{2}\right\rangle=0.8775(51) \mathrm{fm}^{2}[38]$ and $\left\langle R_{n}^{2}\right\rangle=-0.1149(27) \mathrm{fm}^{2}$ [39]. In the present calculations, the contributions of the second term of Eq. (6) to the rms radii are $\leqslant 0.03 \mathrm{fm}$ and decrease with the mass number. References $[19,40]$ have considered firstorder corrections to the approximation of $A$ noninteracting propagators - used to calculate this term - and found that it is negligible in most cases as long as fully correlated densities are used. Therefore, we conclude that Eq. (6) does not introduce sizable errors.

The exact one-body propagator, $g_{\alpha \beta}(\omega)$, also allows calculating the total energy by means of the extended Koltun sum rule [41]:

$$
E_{0}^{A}=\sum_{\alpha \beta} \frac{1}{2} \int_{-\infty}^{\varepsilon_{0}^{-}}\left[T_{\alpha \beta}+\omega \delta_{\alpha \beta}\right] S_{\beta \alpha}^{h}(\omega) d \omega-\frac{1}{2}\langle W\rangle .
$$

This requires only the additional evaluation of the expectation value of the $3 N$ interaction, $\langle W\rangle$. Again, we approximate this in terms of noninteracting three-body density matrices:

$$
\langle W\rangle \simeq \frac{1}{6} \sum_{\alpha \beta \mu \gamma \delta v} W_{\alpha \beta \mu, \gamma \delta \nu} \rho_{\gamma \alpha} \rho_{\delta \beta} \rho_{\nu \mu}
$$

The errors in this approximation have been estimated in Ref. [19] and were found to not exceed $250 \mathrm{keV}$ on the total binding energy for ${ }^{16} \mathrm{O}$ and ${ }^{24} \mathrm{O}$.

In all simulations below we subtract the spurious contribution of the kinetic energy of the c.m. and work with the intrinsic Hamiltonian $H[A]=H-T_{\text {c.m. }}(A)=U(A)+$ $V(A)+W$, which acquires a dependence on total number of nucleons. The $U, V$, and $W$ label one-, two-, and three-body interactions. This implies that the particle and hole spectra of the even-odd isotopes are recalculated separately from $H[A+1]$ and $H[A-1]$. They are then corrected for the c.m. motion as

$$
\begin{aligned}
& \varepsilon_{n, \mathrm{c} . \mathrm{m} .}^{+}=\varepsilon_{n}^{+}[A+1]+E_{0}^{A}[A+1]-E_{0}^{A}[A], \\
& \varepsilon_{k, \mathrm{c} . \mathrm{m} .}^{-}=\varepsilon_{k}^{-}[A-1]-E_{0}^{A}[A-1]+E_{0}^{A}[A],
\end{aligned}
$$

where $\varepsilon_{n}^{ \pm}[A \pm 1]$ and $E_{k}^{A}[A \pm 1]$ label the poles of $g_{\alpha \beta}(\omega)$ and the total energies, Eq. (10), calculated from the $H[A \pm 1]$ Hamiltonian. The overall c.m. corrections become progressively smaller as $A$ increases.

\section{Dyson-ADC(3) and second-order Gorkov equations}

Calculations with $3 N$ interactions follows the procedure extensively discussed in Ref. [41], which involves defining the following medium-dependent one- and two-body interactions:

$$
\begin{aligned}
\widetilde{U}_{\alpha \beta} & =U_{\alpha \beta}+\sum_{\delta \gamma} V_{\alpha \gamma, \beta \delta} \rho_{\delta \gamma}+\frac{1}{4} \sum_{\mu \nu \gamma \delta} W_{\alpha \mu \nu, \beta \gamma \delta} \rho_{\gamma \mu} \rho_{\delta \nu}, \\
\widetilde{V}_{\alpha \beta, \gamma \delta} & =V_{\alpha \beta, \gamma \delta}+\sum_{\mu \nu} W_{\alpha \beta \mu, \gamma \delta \nu} \rho_{\nu \mu} .
\end{aligned}
$$

This allows neglecting residual contributions in $W$ that have been found to be negligible for oxygen isotopes [42,43]. Hence, we retain only interaction-irreducible diagrams in $\widetilde{U}$ and $\widetilde{V}$ to the self-energy.

To solve Eq. (2), we express the self-energy as

$$
\Sigma_{\alpha \beta}^{\star}(\omega)=\Sigma_{\alpha \beta}(\infty)+\sum_{i j} \boldsymbol{D}_{\alpha i}^{\dagger}\left[\frac{1}{\omega-(\boldsymbol{K}+\boldsymbol{C})}\right]_{i j} \boldsymbol{D}_{j \beta},
$$

where $\Sigma_{\alpha \beta}(\infty)$ is the correlated and energy-independent mean field. The whole $\Sigma_{\alpha \beta}^{\star}(\omega)$ is an optical potential for elastic scattering of a nucleon off the $\left|\Psi_{0}^{A}\right\rangle$ ground state, which also describes the fragmentation of the particle and hole spectra [44,45].

In Eq. (14), the matrix $\mathbf{D}$ couples single-particle states to more complex intermediate configurations, while $\mathbf{K}$ and $\mathbf{C}$ are their unperturbed energies and interaction matrices. For the closed subshell isotopes we exploit the third-order algebraic diagrammatic construction [ADC(3)] scheme, which is the best compromise between computational efforts and accuracy. This consists of the minimal choice of these matrices that retains all self-energy diagrams up to third order. Although $\mathrm{ADC}(3)$ is constrained at third order, it contains infinite order summations of diagrams that include particle-particle and hole-hole ladders as well as particle-hole rings. It is therefore a fully nonperturbative approach. Generally speaking, $\operatorname{ADC}(n)$ defines a hierarchy of truncation schemes of Eq. (14) for increasing order $n$ that allows systematic improvements of the method [46].

Recently, SCGF theory has been extended to a NambuGorkov formulation that allows addressing truly open-shell nuclei [29]. This has opened the possibility to calculate ground-state properties and the one-nucleon addition and removal spectra of midmass open-shell nuclei in a fully $a b$ initio fashion. As in BCS theory, one allows for an explicit breaking of particle-number conservation that is necessary for a proper description of pairing correlations [29,47]. This implies introducing a grand-canonical Hamiltonian $\Omega=H-$ $\mu_{n} \hat{N}-\mu_{p} \hat{Z}$ and constraining the proton (neutron) chemical potentials $\mu_{p}\left(\mu_{n}\right)$ to recover the correct particle number on average, $A=\left\langle\Psi_{0}|\hat{A}| \Psi_{0}\right\rangle$, where $\left|\Psi_{0}\right\rangle$ is the symmetry-broken ground state. A detailed description of the theory can be found in Refs. [29,30,48]. 
In Gorkov theory one is left with a set of normal and anomalous propagators and self-energies with similar Lehman representations to Eqs. (1) and (14). In particular, the normal propagator is

$$
G_{\alpha \beta}^{11}(\omega)=\sum_{k}\left\{\frac{\mathcal{U}_{\alpha}^{k} \mathcal{U}_{\beta}^{k *}}{\omega-\omega_{k}+i \eta}+\frac{\overline{\mathcal{V}}_{\alpha}^{k *} \overline{\mathcal{V}}_{\beta}^{k}}{\omega+\omega_{k}-i \eta}\right\},
$$

where $\mathcal{U}, \mathcal{V}$ are the transition amplitudes for reaching the states $\left|\Psi_{k}\right\rangle$ by adding (removing) a nucleon to (from) $\left|\Psi_{0}\right\rangle$, and $\omega_{k}$ are the corresponding quasiparticle energies [29].

The Gorkov version of the SCGF approach makes it possible to calculate spectral functions for open-shell semimagic systems. Its present formulation follows the $\mathrm{ADC}(n)$ truncation scheme discussed above but has been implemented only up to the second order. This has allowed successful predictions of trends in binding energies [22]. However, the $\mathrm{ADC}(2)$ is known to slightly underestimate binding energies and it is not guaranteed to provide accurate predictions for one-nucleon removal and addition energies [49], which are instead possible with a Dyson-ADC(3) calculations. The full extension to Gorkov-ADC(3) formalism is currently under way [50].

\section{CALCUlations}

Calculations have been performed using $N N$ and $3 N$ chiral interactions evolved to a low-momentum scale $\lambda_{\text {SRG }}$ through free space similarity renormalization group (SRG) techniques [28]. The original $N N$ interaction is the next-to-next-to-nextto-leading order (N3LO) with a cutoff $\Lambda_{N N}=500 \mathrm{MeV} / c$, from Refs. [51,52]. For the $3 N$ interactions we used the NNLO with a reduced local cutoff of $\Lambda_{3 N}=400 \mathrm{MeV} / c$ [43,53]. This includes the two-pion exchange contribution that was originally proposed by Fujita and Miyazawa [54]. Low-energy constants were set at $c_{D}=-0.2, c_{E}=0.098$ to reproduce the ${ }^{3} \mathrm{H} \beta$ decay and the binding energy of ${ }^{4} \mathrm{He}$. With this choice, the binding energy of ${ }^{3} \mathrm{H}$ is $-8.32 \mathrm{MeV}$, to be compared to the experimental value of $-8.48 \mathrm{MeV}$. When we perform the SRG transformation of the sole $N N-\mathrm{N} 3 \mathrm{LO}$ interaction we already obtain evolved $N N+3 N$ interactions. We refer to this as the "induced" Hamiltonian. Conversely, the "full" Hamiltonian is the one obtained by also evolving the original 3NF-NNLO. Therefore, the effects of 3NFs of the Fujita-Miyazawa type are included in the full Hamiltonian only.

All calculations were performed in a model space of 12 harmonic oscillator (HO) shells $\left[N_{\max } \equiv \max (2 n+l)=\right.$ 11], including all $N N$ matrix elements and limiting $3 \mathrm{NF}$ ones to configurations with $N_{1}+N_{2}+N_{3} \leqslant N_{\max }^{3 \mathrm{NF}}=14$. We checked that increasing $N_{\max }^{3 \mathrm{NF}}$ from 14 to 16 changes Gorkov total binding energies by $\approx 500 \mathrm{keV}$. Changing the oscillator frequency between $\hbar \Omega_{\mathrm{HO}}=20$ and $24 \mathrm{MeV}$ in Dyson-ADC(3) calculations, we found up to $450 \mathrm{keV}$ variations in the binding energy of ${ }^{24} \mathrm{O}$. Similarly, varying $\lambda_{\mathrm{SRG}}$ in a limited range $1.88-2.0 \mathrm{fm}^{-1}$ did not induce variations of more than $0.5 \%$. From these and other tests we infer a conservative theoretical error of at most $5 \%$, for binding energies obtained with DysonADC(3) [19]. Similar conclusions can be drawn about the prediction of dominant quasiparticle peaks in the single-particle spectrum, $\varepsilon_{k}^{+}$and $\varepsilon_{n}^{-}$. Varying both $\hbar \Omega_{\mathrm{HO}}=20-24 \mathrm{MeV}$ and $\lambda_{\mathrm{SRG}}=1.88-2.0 \mathrm{fm}^{-1}$, we found a maximum variation of $310 \mathrm{keV}$ for the neutron $1 / 2^{-}$quasihole in ${ }^{24} \mathrm{O}$. This corresponds to $2 \%$ of its value, $\varepsilon_{\nu 1 / 2^{-}}^{-}=-14.22 \mathrm{MeV}$. The largest variation for proton quasiparticle energies was found to be of $550 \mathrm{keV}$ for a $5 / 2^{+}$quasiparticle, mostly owing to variations in $\hbar \Omega_{\mathrm{HO}}$. Therefore, we estimate theoretical errors of $\leqslant 1 \mathrm{MeV}$ for the Dyson-ADC(3) gaps discussed below. For Gorkov calculations, we expect that errors on binding energies and quasiparticle peaks will be larger owing to the simpler many-body truncation. However, we note that Ref. [55] has reported a remarkable independence of dominant quasiparticle peaks on the $\lambda_{\text {SRG }}$ cutoff already at second order.

In the following sections, we report the results obtained for $\hbar \Omega_{\mathrm{HO}}=24 \mathrm{MeV}$ and $\lambda_{\mathrm{SRG}}=2 \mathrm{fm}^{-1}$.

\section{RESULTS}

\section{A. Spectral functions and evolution of single-particle spectra}

Three dimensional plots of the full spectral function, Eq. (3), are illustrated in the Appendix . Here we focus on the energy distribution of the spectral strength calculated by integrating its diagonal part over the single-particle degrees of freedom,

$$
\begin{aligned}
S(\omega)= & \sum_{\alpha} S_{\alpha \alpha}^{p}(\omega)+S_{\alpha \alpha}^{h}(\omega) \\
= & \sum_{n} S F_{n}^{+} \delta\left(\omega-E_{n}^{A+1}+E_{0}^{A}\right) \\
& +\sum_{n} S F_{k}^{-} \delta\left(\omega-E_{0}^{A}+E_{k}^{A-1}\right),
\end{aligned}
$$

which yields the energy distribution of spectroscopic factors. Each peak corresponds to the eigenstate of a neighboring oddeven isotope, whose energy is directly observed in nucleon addition and removal experiments.

The particle and hole contributions to Eq. (16), calculated with Dyson-ADC(3), are displayed in Fig. 1 for protons and in Fig. 2 for neutrons. The nucleon addition part of the spectra are highlighted by the shaded areas. These figures show the general features of the correlated spectral distribution, which conserves strong quasiparticle fragments close to the Fermi surface but becomes heavily fragmented as one moves further away owing to coupling to $2 \mathrm{p} 1 \mathrm{~h}$ and $2 \mathrm{~h} 1 \mathrm{p}$ (or more complex) excitations. Quasiparticle states with positive energies are above the one-nucleon continuum threshold $\left(E_{n}^{A+1}-E_{0}^{A}=0 \mathrm{MeV}\right)$ and therefore represent states for scattering of a nucleon off the $\left|\Psi_{0}^{A}\right\rangle$ target. Because we assume a discrete model space in our calculations the associated particle continuum is found to be discretized in several peaks that become more dense with increasing energy, reflecting the changes in the density of states for $\left|\Psi_{n}^{A+1}\right\rangle$. Quasihole fragments at large negative energies correspond to highly excited $\left|\Psi_{k}^{A-1}\right\rangle$ states and also display a continuum portion of the spectrum. However, the spectral strength for nucleon removal is less pronounced. This is attributable to the fewer degrees of freedom available to generate $2 \mathrm{~h} 1 \mathrm{p}$ configurations, and it can be equivalently explained in terms of the small overlap between the 


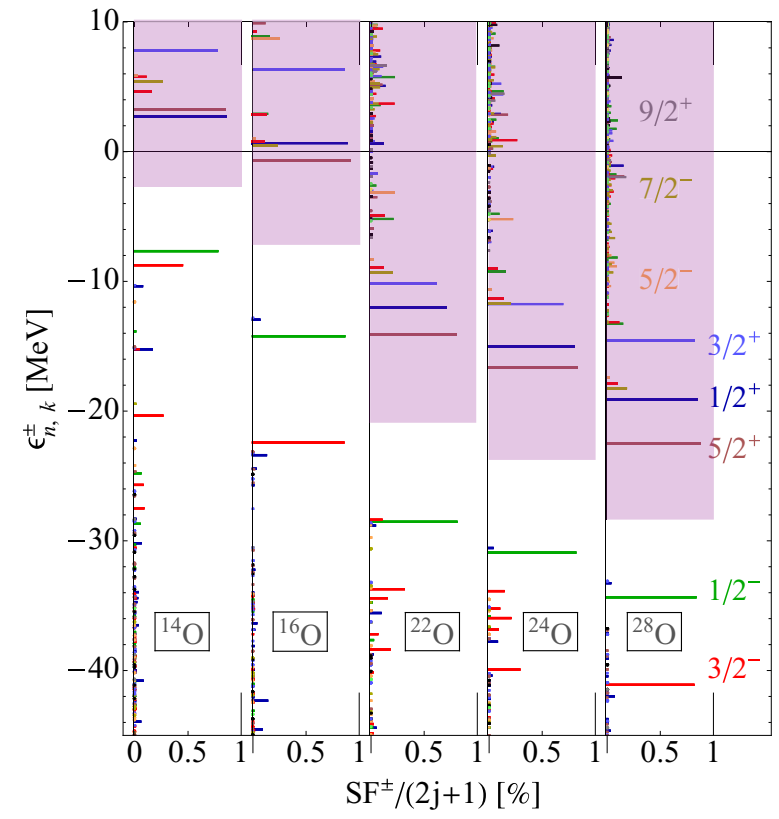

FIG. 1. (Color online) Single-particle spectral distributions for the addition and removal of a proton to/from closed-subshell oxygen isotopes. States above the Fermi surface $\left(E_{F}\right)$ are indicated by the shaded areas and yield the spectra of the resulting odd-even fluorine isotopes. The spectra below $E_{F}$ are for odd-even nitrogen isotopes in the final state (this appears inverted in the plot, with higher excitation energies pointing downward). Fragments with different angular momentum and parity are shown with different colors, as indicated, and the bar lengths provide the calculated spectroscopic factors. These results are obtained from $\mathrm{ADC}(3)$ and the full $N N+3 \mathrm{NF}$ interaction with $\lambda_{\mathrm{SRG}}=2.0 \mathrm{fm}^{-1}$.

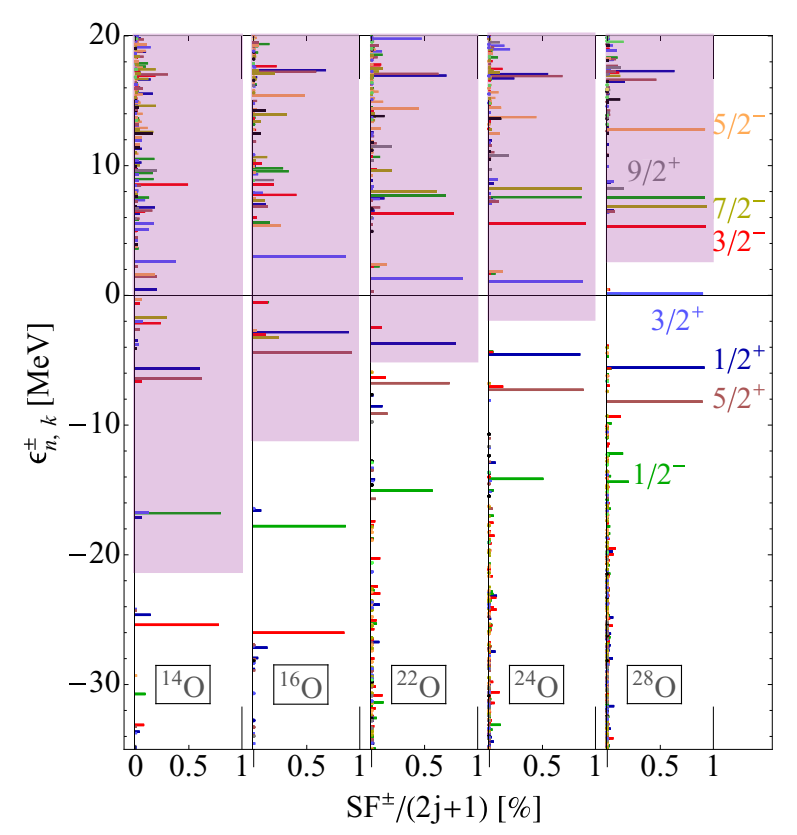

FIG. 2. (Color online) Same as Fig. 1 but for the addition and removal of a neutron. Both particle (shaded areas) and hole spectra correspond to odd-even oxygen isotopes.
$(A-1)$-nucleon wave functions in the continuum and the bound $\left|\Psi_{0}^{A}\right\rangle$ ground state.

The fragments of the spectral distribution provide the excitation spectrum for the neighboring odd-even isotopes. For example, the two dominant quasihole peaks in ${ }^{24} \mathrm{O}$ in Fig. 2 correspond to the $1 / 2^{+}$ground state and the $5 / 2^{+}$ excitation of ${ }^{23} \mathrm{O}$. Our calculated excitation energy for the $5 / 2^{+}$state is $2.74 \mathrm{MeV}$, close to the experimental value of 2.79(13) $\mathrm{MeV}$ [64]. The $3 / 2^{+}$state of ${ }^{23} \mathrm{O}$ can be calculated from the quasiparticle spectra of ${ }^{22} \mathrm{O}$. For this we obtain 5.0 MeV excitation energy, which is larger than the experimental value of $4.0 \mathrm{MeV}$ [62]. In both cases, the theoretical result agrees with the ab initio configuration interaction (CI) calculations of Refs. [32,33], which use the same $N N+3 \mathrm{NF}$ full Hamiltonian. As mentioned above, satellite peaks (that is, nondominant ones) are not necessarily well described in nucleon-attached and nucleon-removal methods at the ADC(3) level. This because they require leading-order configurations of $2 \mathrm{p} 1 \mathrm{~h} / 2 \mathrm{~h} 1 \mathrm{p}$ type or higher. The first $1 / 2^{+}$excited state of ${ }^{21} \mathrm{O}$, seen as a hole on ${ }^{22} \mathrm{O}$, is of this type and has a spectroscopic factor $\approx 9 \%$ of the independent particle model. In spite of this, the $\mathrm{ADC}(3)$ excitation energy is $1.78 \mathrm{MeV}$, which is again in great agreement with CI calculations based on the same Hamiltonian (and slightly off the experimental value of $1.22 \mathrm{MeV}$ [65]). Instead, the calculated spectroscopic factor the the $3 / 2^{+}$ excited state is only $<1 \%$ and this is unlikely to be converged with respect to the many-body truncation in the ADC(3). For this state, we obtain an excitation energy of $940 \mathrm{keV}$ that disagrees with both the experiment and the ab initio CI results, as expected. These results give a further confirmation of the performance of the present chiral Hamiltonian with the single $s d$ shell. Furthermore, we note that the comparison with Refs. $[32,33]$ provides a successful benchmark of the accuracy of $\mathrm{ADC}(3)$ for calculating dominant quasiparticle states. We then use the latter to discuss the single-particle structure across both $p$ and $s d$ shells.

Figure 3 shows the details of the evolution of the dominant proton quasiparticle and quasihole peaks in the $s d$ and $p$ shells for increasing neutron number. These are corrected for the effects of the c.m. motion according to Eqs. (12). The dashed lines are obtained from the $N N+3 N$-induced interaction and represent the spectrum predicted by the initial N3LO $N N$ force. In general, the addition of original 3NFs (solid lines) has the effect of consistently increasing the spin-orbit splittings between the $1 / 2^{-}-3 / 2^{-}$and the $3 / 2^{+}-5 / 2^{+}$dominant peaks. The $s_{1 / 2}$ orbit remain largely unaffected. The overall changes introduced by leading-order $3 \mathrm{NFs}$ are reported in Tables I and II for both protons and neutrons. The evolution of quasiparticle energies for the addition and the removal of a neutron is displayed in Fig. 4. In this case, the $1 / 2^{-}$and $3 / 2^{-}$strength (in the $p$ shell) is strongly fragmented for masses above $A=20$ and no clear dominant peak is predicted. The original 3 NFs still have the effect of increasing the splitting between spin-orbit partner states. However, this is in addition to the stronger repulsion on the $d_{3 / 2}$ orbit that is at the origin of the anomalous dripline at ${ }^{24} \mathrm{O}[16]$.

Worth mentioning are the splittings between the $1 / 2^{-}$and the $3 / 2^{-}$quasiholes in ${ }^{16} \mathrm{O}$. For protons, this is predicted to be 


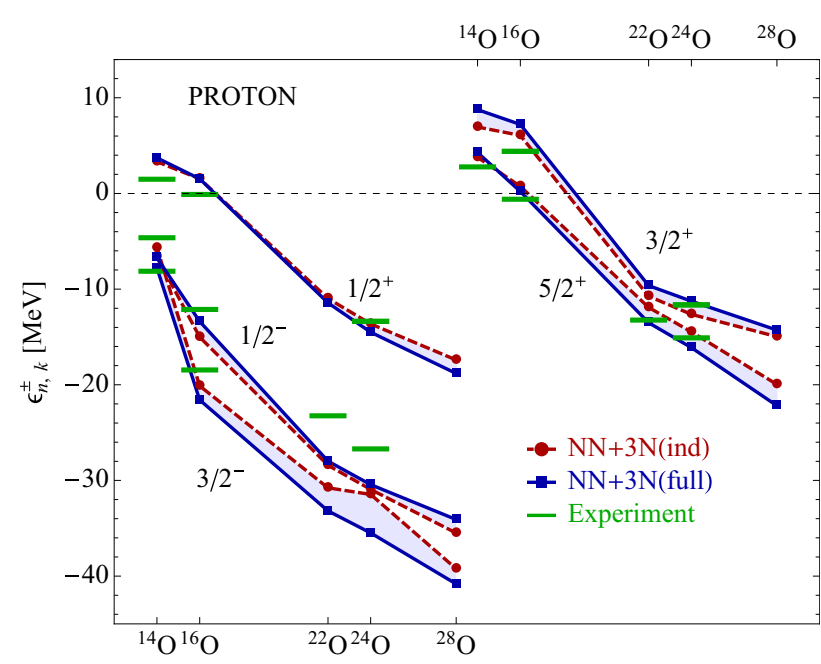

FIG. 3. (Color online) Energy evolution of dominant proton quasiparticle fragments around oxygen isotopes of increasing neutron numbers. These level are for protons in the $p$ and $s d$ shells and refer to the ground and excited states of odd-even nitrogen $\left(1 / 2^{-}, 3 / 2^{-}\right)$ and fluorine $\left(5 / 2^{+}, 1 / 2^{+}, 3 / 2^{+}\right)$. Dots (joined by dashed lines) show $\mathrm{ADC}(3)$ results obtained with the induced $N N+3 \mathrm{NF}$ interaction. Squares (with solid lines) refer to the full Hamiltonian, with the leading NNLO-3NF included. The latter are the dominant peaks also displayed in Fig. 1. In all cases $\lambda_{\mathrm{SRG}}=2.0 \mathrm{fm}^{-1}$. Experimental values are from Refs. [56-59].

$5.1 \mathrm{MeV}$ by the $N N+3 N$-induced interaction, which is close to the empirical value of $6.32 \mathrm{MeV}$. However, the full Hamiltonian increases it to $8.2 \mathrm{MeV}$, overestimating the experiment. Exactly the same situation is found for the splitting between corresponding neutron holes, which is also increased by 3.1 $\mathrm{MeV}$ owing to the original $3 \mathrm{NF}$ at NNLO. For comparison, the Argonne v18 interaction that has a strongly repulsive core predicts a separation of $\approx 3.1 \mathrm{MeV}$ [66] for these two states, at the $N N$ interaction level. The corresponding Urbana IX $3 \mathrm{NF}$ increases this by another $2.7 \mathrm{MeV}$ predicting a splitting that is much closer to the experiment [67]. Both $3 N$ Hamiltonians include two-pion terms of the Fujita-Miyazawa type and it is therefore reasonable that they generate similar corrections.

From Tables I and II it is clear that the present $N N+3 N$ chiral Hamiltonians have a slight tendency to stretch the
TABLE II. Spin-orbit splittings between $1 / 2^{-}$and $3 / 2^{-}$quasiparticle fragments. For the case of protons these are eigenstates of the odd-even ${ }^{A-1} \mathrm{~N}$ isotopes indicated in the second row. For neutrons, they are states of odd-even ${ }^{A-1} \mathrm{O}$, except for ${ }^{14} \mathrm{O}$, where the two quasiparticles correspond to different isotopes. Results are reported for both the $N N+3 N$-induced and full Hamiltonians and $\Delta \varepsilon_{p L S}=\varepsilon_{p L S}^{\mathrm{FULL}}-\varepsilon_{p L S}^{\mathrm{IND}}$ are the contributions owing to original $3 \mathrm{NFs}$ at NNLO. Experimental values are from Refs. [56,58].

\begin{tabular}{|c|c|c|c|c|c|c|c|}
\hline \multirow[t]{2}{*}{$\varepsilon_{p L S}=\varepsilon_{1 / 2^{-}}-\varepsilon_{3 / 2^{-}}$} & \multicolumn{5}{|c|}{ Protons } & \multicolumn{2}{|c|}{ Neutrons } \\
\hline & ${ }^{13} \mathrm{~N}$ & ${ }^{15} \mathrm{~N}$ & ${ }^{21} \mathrm{~N}$ & ${ }^{23} \mathrm{~N}$ & ${ }^{27} \mathrm{~N}$ & $15,13 \mathrm{O}$ & ${ }^{15} \mathrm{O}$ \\
\hline$\Delta \varepsilon_{p L S}$ & 2.00 & 3.10 & 2.84 & 4.52 & 3.01 & 3.66 & 3.01 \\
\hline$\varepsilon_{p L S}^{\mathrm{IND}}$ & -0.92 & 5.10 & 2.36 & 0.53 & 3.73 & 5.05 & 5.16 \\
\hline$\varepsilon_{p L S}^{\mathrm{FULL}}$ & 1.07 & 8.21 & 5.20 & 5.05 & 6.74 & 8.71 & 8.24 \\
\hline$\varepsilon_{p L S}^{\exp }$ & 3.50 & 6.32 & & & & 9.95 & 6.18 \\
\hline
\end{tabular}

single-particle spectrum, as compared to the experimentally observed dominant peaks. Corrections to these flaws may come at the price of introducing extra short-range repulsion in the $N N$ interactions, for example through higher chiral cutoffs. At lower resolution scales, this implies the possible presence of relevant many-body forces at least at the $4 \mathrm{NF}$ level. Reference [22] pointed out that the experimental gaps between the $s d$ and $p f$ shells are overestimated for the $\mathrm{Ca}$ and neighboring isotopic chains. To investigate the behavior in the present case, we consider the separations between the dominant $5 / 2^{+}$and $1 / 2^{-}$fragments that is representative of the gap between the $p$ and $s d$ shells. These are reported in Table III. With the only exception of the proton gap in ${ }^{14} \mathrm{O}$, we find that preexisting $3 \mathrm{NFs}$ have the effect of reducing the distance between the two shells by about $2 \mathrm{MeV}$ and bringing it closer to the experiment. In spite of this, the gaps remain consistently predicted too large by just a few $\mathrm{MeV}$ even when the full Hamiltonian is used.

\section{B. Spectroscopic factors}

The quenching of spectroscopic factors (SFs) for the dominant quasiparticle peaks can provide useful insights on the strength of the correlations generated by the Hamiltonian. The principal mechanisms that are responsible for these are identified with the coupling of nucleons to high-momentum

TABLE I. Spin-orbit splittings between $3 / 2^{+}$and $5 / 2^{+}$quasiparticle fragments. For the case of protons these are eigenstates of the odd-even ${ }^{A+1} \mathrm{~F}$ isotopes indicated in the second row. In the case of neutrons, they are states of odd-even ${ }^{A \pm 1} \mathrm{O}$. Note that for neutrons and $A=22,24$, these two levels are found across the Fermi surface and correspond to eigenstates of different isotopes. Results are reported for both the $N N+3 N$-induced and full Hamiltonians and $\Delta \varepsilon_{d L S}=\varepsilon_{d L S}^{\mathrm{FULL}}-\varepsilon_{d L S}^{\mathrm{IND}}$ are the changes owing to adding the original $3 \mathrm{NFs}$ at NNLO. Experimental values are from Refs. [56,57,59-63].

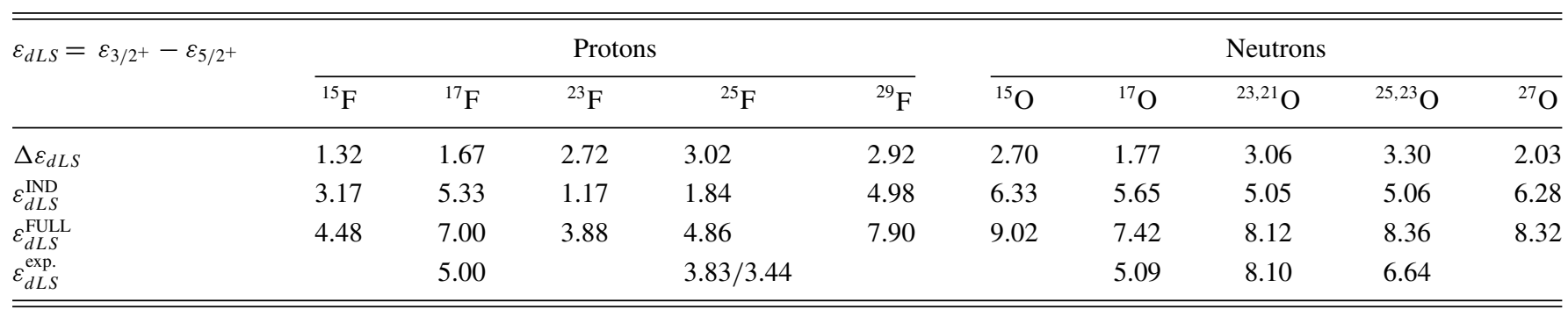




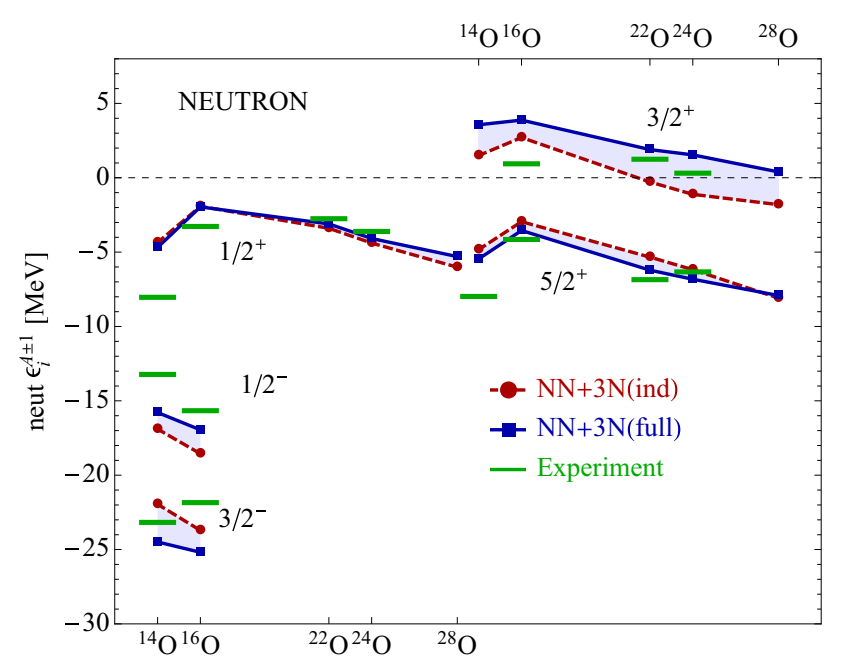

FIG. 4. (Color online) Same as Fig. 3 but for dominant neutron fragments. Dots (joined by dashed lines) shows $\mathrm{ADC}(3)$ results obtained with the induced $N N+3 N F$ interaction. Squares (with full lines) refer to the full Hamiltonian and the same shown in Fig. 2. Experimental values are from Refs. [56,57,60-63].

states owing to short-range physics and with long-range effects that include collective resonance modes and configuration mixing at small excitation energies [35]. For short-range correlations, we refer to the effects of the repulsive part of the central and tensor $N N$ interactions, typically at distances $<1 \mathrm{fm}$, traditionally used to reproduce nuclear phase shifts at very high energies. Even for Hamiltonians that present strongly repulsive $N N$ cores, the effects of short-range physics is usually found to be at most a $10 \%$ reduction with respect to the independent particle model prediction [1]. Thus, the quenching of SFs is mostly a consequence of low-energy physics. For the present Hamiltonian, the SRG evolution completely removes any quenching owing to short-range correlations.

TABLE III. Energy gaps between the dominant $5 / 2^{+}$and $1 / 2^{-}$ quasiparticles. These give a measure of the gaps between the $s d$ and $p$ shells for the ${ }^{A} \mathrm{O}$ isotopes indicated in the second row. For the case of protons, these are particle-hole gaps and coincide with the ground states of the corresponding odd-even ${ }^{A+1} \mathrm{~F}$ and ${ }^{A-1} \mathrm{~N}$ isotopes. For neutrons, these are eigenstates of odd-even ${ }^{A \pm 1} \mathrm{O}$ but are not necessarily situated across the Fermi surface. Results are reported for both the $N N+3 N$-induced and full Hamiltonians and $\Delta E_{\text {gap }}=E_{\text {gap }}^{\mathrm{FULL}}-E_{\text {gap }}^{\mathrm{IND}}$ are the effects of the original $3 \mathrm{NFs}$ at NNLO. Experimental data are from Refs. [56-61,63].

\begin{tabular}{|c|c|c|c|c|c|c|c|}
\hline \multirow{2}{*}{$\begin{array}{l}E_{\text {gap }} \\
=\varepsilon_{5 / 2^{+}}^{A+1} \\
-\varepsilon_{1 / 2^{-}}^{A^{-}}\end{array}$} & \multicolumn{5}{|c|}{ Protons } & \multicolumn{2}{|c|}{ Neutrons } \\
\hline & ${ }^{14} \mathrm{O}$ & ${ }^{16} \mathrm{O}$ & ${ }^{22} \mathrm{O}$ & ${ }^{24} \mathrm{O}$ & ${ }^{28} \mathrm{O}$ & ${ }^{14} \mathrm{O}$ & ${ }^{16} \mathrm{O}$ \\
\hline$\Delta E_{\text {gap }}$ & 0.61 & -2.16 & -2.03 & -2.14 & -3.64 & -1.74 & -2.20 \\
\hline$E_{\text {gap }}^{\mathrm{IND}}$ & 10.38 & 15.76 & 16.50 & 16.46 & 15.54 & 12.07 & 15.60 \\
\hline$E_{\text {gap }}^{\text {FULL }}$ & 10.99 & 13.60 & 14.47 & 14.32 & 11.90 & 10.32 & 13.40 \\
\hline$E_{\text {gap }}^{\text {exp. }}$ & 7.41 & 11.53 & 10.02 & 13.33 & & 5.24 & 11.52 \\
\hline
\end{tabular}

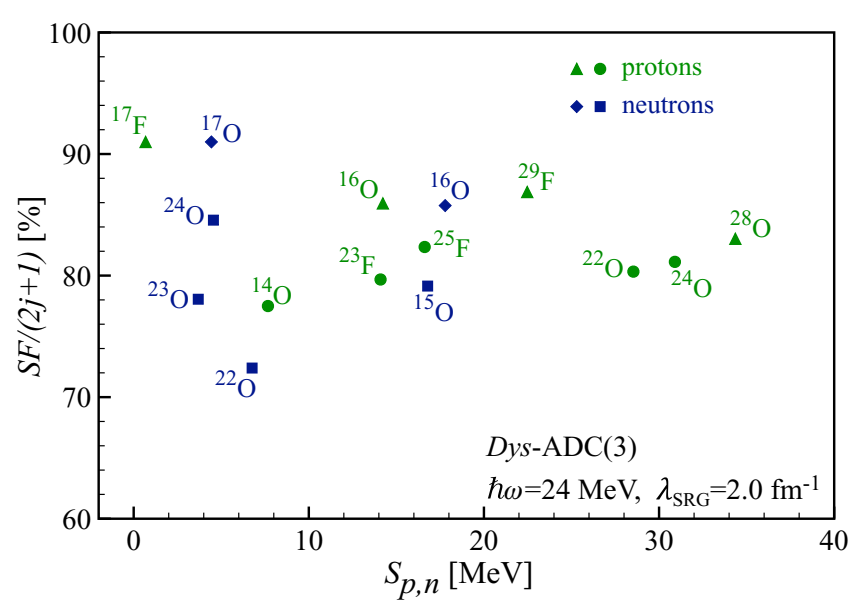

FIG. 5. (Color online) Calculated spectroscopic factors plotted as a function of their separation energies. All values shown are for transitions between two ground states and refer to the removal of a proton (blue squares and diamonds) or a neutron (green circles and triangles) from the isotope indicated nearby. Triangles and diamonds highlight transitions that involve the ${ }^{16} \mathrm{O}$ and ${ }^{28} \mathrm{O}$ isotopes at major shell closures. These results are for $\mathrm{ADC}(3)$ and the full $N N+3 \mathrm{NF}$ interaction.

Because SFs are directly linked to the cross sections probed by particle addition and removal processes, it has long been debated whether their evolution with proton-neutron asymmetry can explain the observed variations in the strength of direct nucleon knockout cross sections $[68,69]$. The difference between the proton and neutron separation energies is normally taken as a measure of such asymmetry. A case of particular interest is ${ }^{14} \mathrm{O}$ because of the very large value of this quantity. The present ADC(3) calculations yield substantially the same SFs equal to $77.4 \%(77.2 \%)$ for the removal of a proton (neutron) from this isotope to the ground state of ${ }^{13} \mathrm{~N}\left({ }^{13} \mathrm{O}\right)$. Recent measurements of the $\left(d,{ }^{3} \mathrm{He}\right)$ and $\left(d,{ }^{3} \mathrm{H}\right)$ reactions are found to be consistent with our calculations and therefore support a near independence of correlations effects from proton and neutron separation energies [70].

To extend the analysis to cases with larger differences between proton and neutron numbers, we plot in Fig. 5 the SFs for ground-state-to-ground-state transitions along the whole chain. In general, we find values evenly spread between $70 \%$ and $90 \%$ of the independent particle model. The smaller values of SFs are obtained at low separation energies and involve transitions to/from ${ }^{14,22,24} \mathrm{O}$. These isotopes present reduced particle-hole neutron gaps and therefore allow for stronger correlations at the Fermi surface. This consideration is also consistent with previous works that clearly showed a close correlations between the particle-hole gap at the Fermi surface and the predicted values of SFs [27]. From this, one may infer that the over stretched spectra reported in Tables I, II, and III result in more modest quenchings of SFs than otherwise expected.

By looking only at transitions that involve the doubly closed major shells ${ }^{16} \mathrm{O}$ and ${ }^{28} \mathrm{O}$, one can still identify a correlation between SFs and nucleon separation energies. In particular, proton orbits tend to be more deeply bound as 
the number of neutrons increases. This is attributable to the strong components of the proton-neutron forces, which also enhances their correlations. However, the overall dependence on proton-neutron asymmetry is rather mild. We note that the vicinity to the neutron dripline would require to explicitly account for the continuum. Reference [71] found that this effect is sizable for ${ }^{24,28} \mathrm{O}$ and leads to further quenching of the proton SFs. Again, this could be interpreted as a reduced gap between the highest neutron quasihole state and the nearby particle continuum. In this sense, the reduction of SFs is an indirect consequence of the change in proton-neutron asymmetry, which first affects energy gaps.

For the case of the $N N+3 N$-induced Hamiltonian we find a completely similar picture, with SFs of dominant peaks being on average slightly larger than those obtained with the full interaction. Also in this case, stronger quenchings are associated with increased fragmentation of nearby strength and the narrowing of (sub-)shell gaps. Thus, we conclude that the general effects of the original 3NFs on the quenching of absolute SFs mainly results from the rearrangement of shell orbits and excitation gaps.

\section{Results for open shells}

The present implementation of the Gorkov-GF approach allows calculations up to the second order in the self-energy [i.e., at the $\operatorname{ADC}(2)$ level]. Although this does not guarantee the best precision for quasiparticle energies [49], it still yields proper predictions for the trend of binding energies [22].

We plot the Gorkov-predicted binding energies for all oxygen isotopes in Fig. 6 and compare them to the DysonADC(3) results where available. For the Dyson case, the $N N+3 N$-induced Hamiltonian systematically underbinds the full isotopic chain and predicts ${ }^{28} \mathrm{O}$ to be bound with

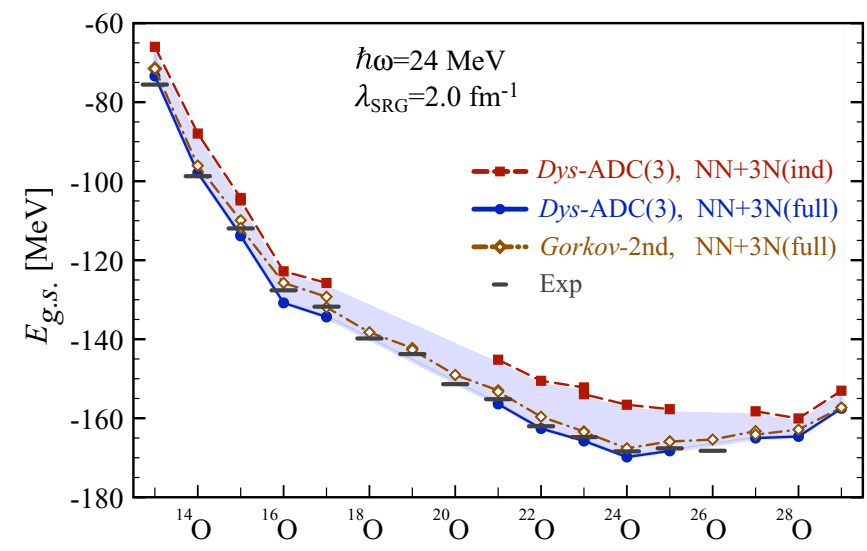

FIG. 6. (Color online) Binding energies of oxygen isotopes. Dashed and solid lines join the results from Dyson-ADC(3) calculations with the $N N+3 N$-induced (squares) and full (circles) Hamiltonians. The shaded area highlights the changes owing to the original $3 \mathrm{NF}$ at NNLO. The open diamonds, joined by dot-dashed lines, are from Gorkov calculations at second order and include open-shell isotopes. Odd-even isotopes are obtained by summing total binging energies of the even-even systems [Eq. (10)] and the energies for addition or removal of a neutron [Eq. (12)]. Experiment are from Refs. [56,57,60,63,72]. respect to ${ }^{24} \mathrm{O}$. This is fully corrected by including the original $3 \mathrm{NF}$ at leading order, which brings all results to about $3 \%$ form the experiment or closer. This is well within the estimated theoretical errors discussed above [19]. The dotdashed line shows the trend of ground-state energies for the full Hamiltonian obtained form Gorkov, which include the ${ }^{18,20,26} \mathrm{O}$ isotopes. This demonstrates that the fraction of binding missed by the second-order truncation is rather constant across the whole isotopic chain and, in the present case, of about 2-4 MeV. The result is a constant shift with respect to the complete $\mathrm{ADC}(3)$ prediction and the overall trend of binding energy is reproduced very close to the experiment. Note that binding energies for odd-even oxygens can be calculated either as neutron addition or neutron removal from two different nearby isotopes. Figure 6 shows that this procedure can lead to somewhat different results, which should be taken as an indication of the errors owing to the second-order many-body truncation. For the more complete Dyson-ADC(3) method and the full Hamiltonian, these differences are never larger than $200 \mathrm{keV}$ and are not visible in the plot. Our calculations with the more accurate Dyson-ADC(3) scheme predict ${ }^{28} \mathrm{O}$ to be unbound with respect to ${ }^{24} \mathrm{O}$ by $5.2 \mathrm{MeV}$. However, this value should be slightly affected by the vicinity to the continuum [17], which was neglected in the present work.

Figure 7 shows the analogous information for the binding energies of the nitrogen and fluorine isotopic chains, obtained through removal and addition of one proton. This confirms that all considerations made regarding the effects of leading-order $3 \mathrm{NFs}$ on the oxygens also apply to their neighboring chains. In particular, the repulsive effect on the $d_{3 / 2}$ neutron orbit is key in determining the neutron driplines at ${ }^{23} \mathrm{~N}$ and ${ }^{24} \mathrm{O}$. Fluorine isotopes have been observed experimentally up to ${ }^{31} \mathrm{~F}$ but with a ${ }^{29} \mathrm{~F}$ that is very weakly bound. Figure 7 clearly demonstrates that this is attributable to an very subtle cancellation between the repulsion form $3 \mathrm{NFs}$ and the attraction generated by one extra proton [19].

The general qualitative features of the spectral functions discussed in the previous sections are also found in our Gorkov propagators but with an even more spread single-particle

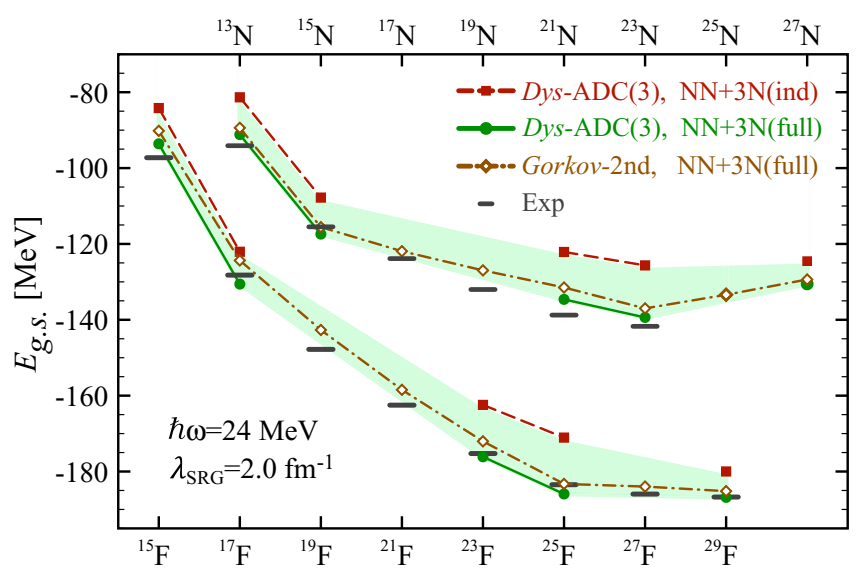

FIG. 7. (Color online) Same as Fig. 6 but for the binding energies of nitrogen and fluorine isotopes. These are calculated as addition or removal of a proton to and from even-even oxygen isotopes. Experiment are from Refs. [56-58,63,72]. 


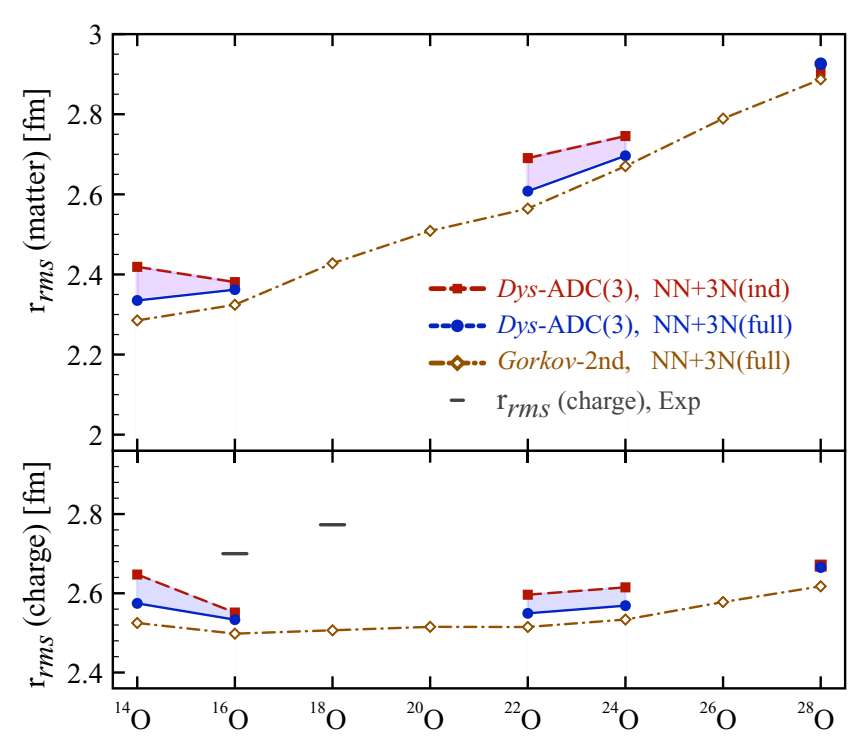

FIG. 8. (Color online) Matter radii (top) and charge radii (bottom) obtained from Dyson-ADC(3) and second-order Gorkov. Curves are the same as in Fig. 6. The bars are experimental charge radii from Ref. [39].

spectra. For example, the splitting among the $1 / 2^{-}$and $3 / 2^{-}$ quasihole states of ${ }^{15} \mathrm{~N}$ is found to be $10.2 \mathrm{MeV}$, compared to the $8.2 \mathrm{MeV}$ calculated in the Dyson-ADC(3) scheme (cf. Table II). This larger value is a consequence of neglecting the interactions between $2 \mathrm{p} 1 \mathrm{~h}$ and $2 \mathrm{~h} 1 \mathrm{p}$ configurations by the second-order truncation. Interestingly, this splitting is sensibly reduced in the neighboring semimagic isotopes and it is calculated to be $4.9 \mathrm{MeV}$ for ${ }^{17} \mathrm{~N}$ and $5.6 \mathrm{MeV}$ for ${ }^{19} \mathrm{~N}$. These values refer to the separation from the first $3 / 2^{-}$state close to the Fermi surface (rather than a centroid of the first few fragments). They are sensibly smaller because the calculations yield a fragmented $p_{3 / 2}$ hole orbit for these nuclei. In the Gorkov calculations, the changes in these splittings owing to aiding the original NNLO-3NF $\left(\Delta \varepsilon_{p L S}\right)$ are 4.2, 0.8, and 2.2 MeV, respectively, for ${ }^{15} \mathrm{~N},{ }^{17} \mathrm{~N}$, and ${ }^{19} \mathrm{~N}$.

Figure 8 demonstrates the trend obtained for point-matter and charge radii along the whole chain and compares them to the observed charge radii for ${ }^{16} \mathrm{O}$ and ${ }^{18} \mathrm{O}$. The induced $3 \mathrm{NFs}$ give sizable contributions to the calculated radii, which would be sensibly smaller if computed from the evolved $N N$-only interaction [73]. Conversely, the original 3NFs cause only a small reduction. Eventually, the radii predicted by the complete Dyson-ADC(3) calculations and the full Hamiltonian are smaller than the experimental charge radii by $0.2-0.3 \mathrm{fm}$. Note that the present calculations do not account for the evolution of operators through the SRG. However, the small radii are consistent with the overstretched spectra discussed above. The radii calculated with the second-order Gorkov approach give somewhat smaller results owing to the many-body truncation. Nevertheless, they describe the overall trend of increasing matter radii along the whole chain, while charge radii remain largely independent of neutron number. Note that the available experimental data show almost equal charge radii for ${ }^{16} \mathrm{O}$ and ${ }^{17} \mathrm{O}$ and a slight larger value for ${ }^{18} \mathrm{O}$ [39]. This is reminiscent of the behavior of isotope shifts in calcium isotopes that first increase and then decrease when going from ${ }^{40} \mathrm{Ca}$ and ${ }^{48} \mathrm{Ca}$, although more data up to ${ }^{22} \mathrm{O}$ would be required to confirm this. The bell shape observed in calcium isotopes is explained by extensive shell-model calculations covering both $s d$ and $p f$ shells $[74,75]$ or by surface vibrations [76]. Such correlations are not included in the present Gorkov formalism at second order and therefore the flat behavior of charge radii of Fig. 8 is consistent with the many-body truncations adopted here.

\section{CONCLUSIONS}

We have presented a comprehensive study of the single-particle spectral functions and ground-state properties of oxygen isotopes based on chiral $N N+3 N$ interactions. Toward this goal, we performed $a b$ initio calculations within SCGF theory. The theoretical framework of this approach has been reviewed highlighting the physics information contained in the nuclear spectral function. Calculations were performed for the closed-subshell isotopes using the Dyson-ADC(3) many-body truncation scheme, which is presently the state-of-the-art technique. For the open-shell isotopes ${ }^{18} \mathrm{O},{ }^{20} \mathrm{O}$, and ${ }^{26} \mathrm{O}$ we performed calculations using the recently introduced Gorkov formulation of SCGF, which can be currently applied at second order.

The general features of the nuclear spectral functions have been discussed, with particular emphasis on the quasiparticle energies (also referred to as "separation" energies) for the dominant peaks observed in the removal and addition of a nucleon. The $3 N$ interactions at NNLO have the effect of increasing the spin-orbit splittings of the $p$ and $d$ orbits and lead to overestimating the experiment. At the same time, the $3 \mathrm{NFs}$ reduce the gaps between the $p$ and $s d$ major shells, improving the agreement with data but not enough to reproduce the empirical values. We observe that all these deficiencies might be corrected by having extra short-range repulsion in the $N N$ section of the Hamiltonian. Other approaches, such as global fittings of chiral $N N+3 N$ forces to include medium-mass isotopes, also hold the promise to reach proper saturation [77]. In general, it is found that the current $N N-\mathrm{N} 3 \mathrm{LO}$ interaction with cutoff at $500 \mathrm{MeV}$, augmented by the local 3NF-NNLO with a 400-MeV local cutoff, tends to stretch the single-particle spectrum too much compared to data. The corresponding predictions for matter radii underestimate the experiment.

The conclusion that the present chiral forces overestimate the gaps between major shells was already pointed out in Ref. [22] for isotopic chains around $\mathrm{Ca}$ and suggests that these saturate nuclear matter at slightly higher densities than the empirical point $[78,79]$. Here we find that hints of the same pathologies are seen also for the oxygen isotopes, in spite of the fact that binding energies are nicely predicted at smaller masses.

The calculated absolute quenchings of SFs change only mildly with proton-neutron asymmetry. This is valid as long as the occupied states in the single-particle spectrum are not near to the continuum. Stronger correlations would instead be generated by smaller particle-hole gaps in the oxygen isotopes with closed subshells. 

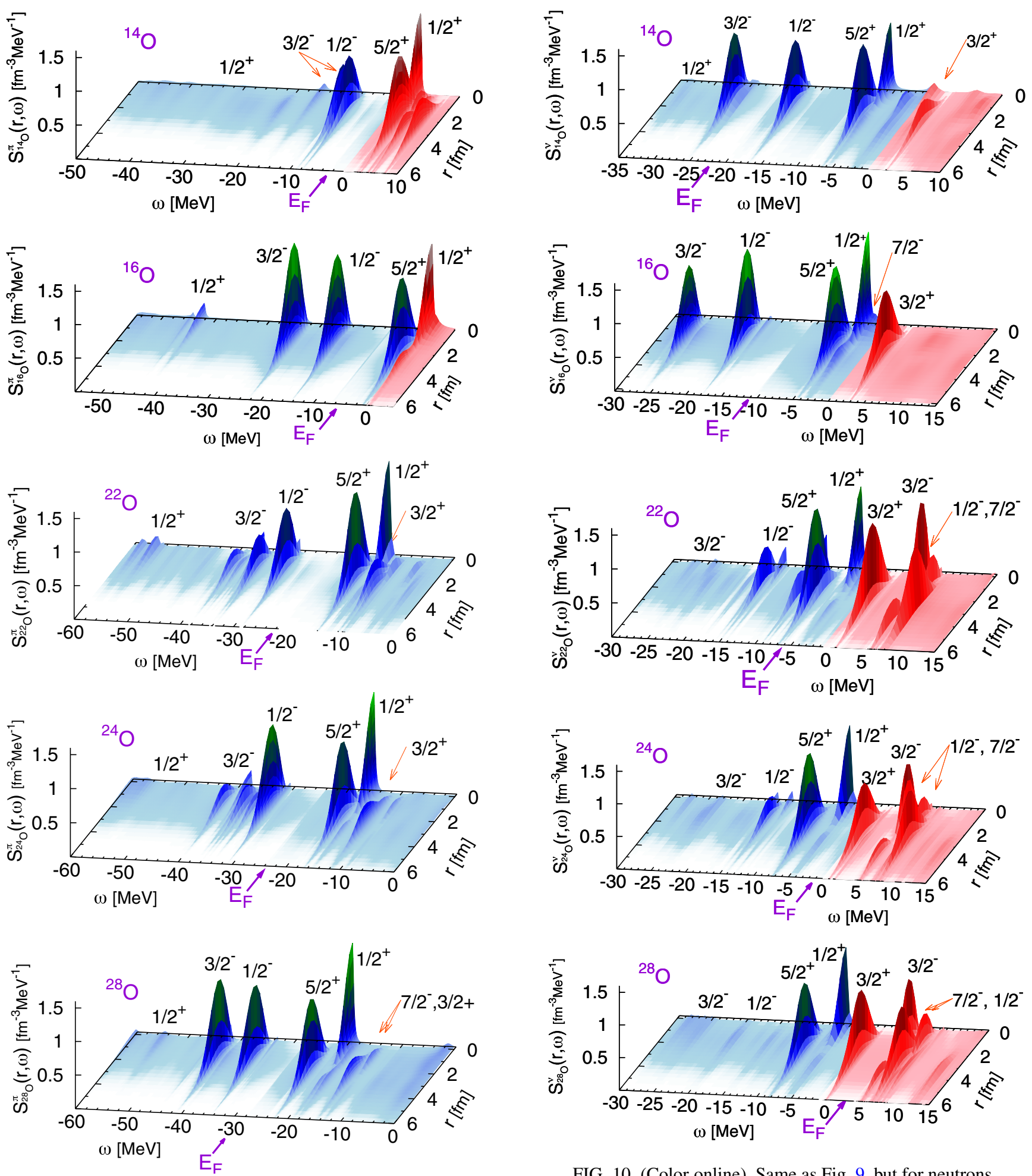

FIG. 10. (Color online) Same as Fig. 9, but for neutrons.

FIG. 9. (Color online) Diagonal part of the complete proton spectral function [Eq. (A1)] for closed-subshell isotopes ${ }^{14,16,22,24,28} \mathrm{O}$. The discretized energy peaks that appear as energy $\delta$ functions in Eq. (3) have been smeared with Lorentzians of suitable with. Energies below the Fermi surface, $E_{F}$, correspond to the hole part of the spectral distribution, while those above are for particle addition. Energies $\omega>0 \mathrm{MeV}$ (plotted in red) correspond to proton-nucleus scattering states.

\section{ACKNOWLEDGMENTS}

The authors would like to thank T. Duguet and V. Somà for their collaboration on developing Gorkov-SCGF codes. This work was supported by the United Kingdom Science and Technology Facilities Council (STFC) under Grants No. ST/J000051/1, No. ST/L005743/1, and No. ST/L005816/1 and 
by the Natural Sciences and Engineering Research Council of Canada (NSERC), Grant No. 401945-2011. Calculations were performed using HPC resources from the DiRAC Data Analytic system at the University of Cambridge (BIS National E-infrastructure Capital Grant No. ST/K001590/1 and STFC Grants No. ST/H008861/1, No. ST/H00887X/1, and No. ST/K00333X/1). TRIUMF receives funding via a contribution through the Canadian National Research Council.

\section{APPENDIX: PLOTS OF SPECTRAL FUNCTIONS}

The diagonal part of the one-nucleon spectral function, Eq. (3), has a straightforward physical interpretation [80,81]. Its particle part, $S_{\alpha \alpha}^{p}(\omega)$, is the joint probability of adding a nucleon with quantum numbers $\alpha$ to the $A$-body ground state, $\left|\Psi_{0}^{A}\right\rangle$, and then to find the system in a final state with energy $E^{A+1}=E_{0}^{A}+\omega$. Likewise, $S_{\alpha \alpha}^{h}(\omega)$ gives the probability of removing a particle from state $\alpha$ and later finding the nucleus in an eigenfunction of energy $E^{A-1}=E_{0}^{A}-\omega$. Once transformed to coordinate or momentum representations, these distributions give a rather intuitive picture of the single-particle structure of a nucleus. We demonstrate this by calculating the spectral function in coordinate basis as

$$
S(r, \omega)=\sum_{\alpha \beta} \phi_{\alpha}(\mathbf{r})\left[S_{\alpha \beta}^{p}(\omega)+S_{\alpha \beta}^{h}(\omega)\right] \phi_{\beta}^{*}(\mathbf{r}),
$$

where $\phi_{\alpha}(\mathbf{r})$ are spin-coupled HO functions. The angular and spin dependencies in Eq. (A1) are removed by summing over all oscillator states. This happens for all nuclei under consideration because they have $J^{\pi}=0^{+}$quantum numbers in their ground states.

The spectral functions obtained from Dyson-ADC(3) calculations are displayed in Figs. 9 and 10 for protons and neutrons, respectively. This shows the radial distribution of the squared one-nucleon overlap wave functions at different quasiparticle energies. The Fermi energy, $E_{F} \equiv\left(\varepsilon_{0}^{+}+\varepsilon_{0}^{-}\right) / 2$, marks the separation between the hole and particle parts of the spectral distribution. Hence, integration over all energies in the range $\omega \in\left[-\infty, E_{F}\right]$ yields the nucleon density $\rho(r)$ [see Eq. (4)] and further integrations over coordinate space yields the particle number. Note that quasiparticle states for $\omega>0$ correspond to the continuum spectrum of the corresponding $(A+1)$-nucleon system. These are unbound states for the scattering of a nucleon off the $\left|\Psi_{0}^{A}\right\rangle$ ground state [44,45]. Thus, they extend to infinity in the limit of a complete singleparticle model space. In the present work, we only calculate their projection on a truncated $\mathrm{HO}$ space, which cannot be normalized to the usual asymptotic boundary conditions. Nevertheless, the plots put in evidence the predicted location for neutron resonances in the $s d$ and $p f$ shells.

It must be kept in mind that these resonances will be further corrected in extended calculations that properly account for the continuum. In general, these effects will be more important the broader is the resonance, and for the present case one may expect corrections as large as a few MeV. Importantly, the selfenergy [Eq. (14)] is a bound function which can be correctly expanded even in a $\mathrm{HO}$ basis. Thus, by first transforming this to coordinate or momentum space, it is possible to obtain a complete optical potential and to compute scattering waves with proper boundary conditions. This is normally done in applications of SCGF theory to scattering $[82,83]$.
[1] W. H. Dickhoff and C. Barbieri, Prog. Part. Nucl. Phys. 52, 377 (2004).

[2] J. Mougey, M. Bernheim, A. Bussière, A. Gillebert, P. X. Hô, M. Priou, D. Royer, I. Sick, and G. Wagner, Nucl. Phys. A 262, 461 (1976).

[3] J. den Herder, H. Blok, E. Jans, P. Keizer, L. Lapikás, E. Quint, G. van der Steenhoven, and P. de Witt Huberts, Nucl. Phys. A 490, 507 (1988).

[4] L. Lapikás, Nucl. Phys. A 553, 297 (1993).

[5] C. J. G. Onderwater et al., Phys. Rev. Lett. 78, 4893 (1997).

[6] D. Rohe et al. (E97-006 Collaboration), Phys. Rev. Lett. 93, 182501 (2004).

[7] C. Barbieri and L. Lapikás, Phys. Rev. C 70, 054612 (2004).

[8] R. Subedi et al., Science 320, 1476 (2008).

[9] D. Middleton et al., Eur. Phys. J. A 29, 261 (2006).

[10] J. P. Schiffer et al., Phys. Rev. Lett. 92, 162501 (2004).

[11] R. V. F. Janssens, Nature (London) 459, 1069 (2009).

[12] F. Wienholtz, D. Beck, K. Blaum, C. Borgmann, M. Breitenfeldt et al., Nature (London) 498, 346 (2013).

[13] D. Steppenbeck et al., Nature (London) 502, 207 (2013).

[14] T. Otsuka, T. Suzuki, R. Fujimoto, H. Grawe, and Y. Akaishi, Phys. Rev. Lett. 95, 232502 (2005).

[15] A. P. Zuker, Phys. Rev. Lett. 90, 042502 (2003).
[16] T. Otsuka, T. Suzuki, J. D. Holt, A. Schwenk, and Y. Akaishi, Phys. Rev. Lett. 105, 032501 (2010).

[17] G. Hagen, M. Hjorth-Jensen, G. R. Jansen, R. Machleidt, and T. Papenbrock, Phys. Rev. Lett. 108, 242501 (2012).

[18] H. Hergert, S. Binder, A. Calci, J. Langhammer, and R. Roth, Phys. Rev. Lett. 110, 242501 (2013).

[19] A. Cipollone, C. Barbieri, and P. Navrátil, Phys. Rev. Lett. 111, 062501 (2013).

[20] J. D. Holt, T. Otsuka, A. Schwenk, and T. Suzuki, J. Phys. G 39, 085111 (2012).

[21] G. Hagen, M. Hjorth-Jensen, G. R. Jansen, R. Machleidt, and T. Papenbrock, Phys. Rev. Lett. 109, 032502 (2012).

[22] V. Somà, A. Cipollone, C. Barbieri, P. Navrátil, and T. Duguet, Phys. Rev. C 89, 061301 (2014).

[23] H. Hergert, S. K. Bogner, T. D. Morris, S. Binder, A. Calci, J. Langhammer, and R. Roth, Phys. Rev. C 90, 041302 (2014).

[24] G. Hagen, T. Papenbrock, M. Hjorth-Jensen, and D. J. Dean, Rep. Prog. Phys. 77, 096302 (2014).

[25] S. Binder, J. Langhammer, A. Calci, and R. Roth, Phys. Lett. B 736, 119 (2014).

[26] K. Tsukiyama, S. K. Bogner, and A. Schwenk, Phys. Rev. Lett. 106, 222502 (2011).

[27] C. Barbieri and M. Hjorth-Jensen, Phys. Rev. C 79, 064313 (2009). 
[28] E. D. Jurgenson, P. Navrátil, and R. J. Furnstahl, Phys. Rev. Lett. 103, 082501 (2009).

[29] V. Somà, T. Duguet, and C. Barbieri, Phys. Rev. C 84, 064317 (2011).

[30] V. Somà, C. Barbieri, and T. Duguet, Phys. Rev. C 87, 011303 (2013).

[31] A. Signoracci, T. Duguet, G. Hagen, and G. Jansen, Phys. Rev. C 91, 064320 (2013).

[32] S. K. Bogner, H. Hergert, J. D. Holt, A. Schwenk, S. Binder, A. Calci, J. Langhammer, and R. Roth, Phys. Rev. Lett. 113, 142501 (2014).

[33] G. R. Jansen, J. Engel, G. Hagen, P. Navratil, and A. Signoracci, Phys. Rev. Lett. 113, 142502 (2014).

[34] J. Mougey, Nucl. Phys. A 335, 35 (1980).

[35] C. Barbieri, Phys. Rev. Lett. 103, 202502 (2009).

[36] H. Hergert and R. Roth, Phys. Lett. B 682, 27 (2009).

[37] J. L. Friar, J. Martorell, and D. W. L. Sprung, Phys. Rev. A 56, 4579 (1997).

[38] P. J. Mohr, B. N. Taylor, and D. B. Newell, Rev. Mod. Phys. 84, 1527 (2012).

[39] I. Angeli and K. Marinova, At. Data Nucl. Data Tables 99, 69 (2013).

[40] C. Barbieri, J. Phys.: Conf. Ser. 529, 012005 (2014).

[41] A. Carbone, A. Cipollone, C. Barbieri, A. Rios, and A. Polls, Phys. Rev. C 88, 054326 (2013).

[42] G. Hagen, T. Papenbrock, D. J. Dean, A. Schwenk, A. Nogga, M. Włoch, and P. Piecuch, Phys. Rev. C 76, 034302 (2007).

[43] R. Roth, S. Binder, K. Vobig, A. Calci, J. Langhammer, and P. Navrátil, Phys. Rev. Lett. 109, 052501 (2012).

[44] F. Capuzzi and C. Mahaux, Ann. Phys. 245, 147 (1996).

[45] J. Escher and B. K. Jennings, Phys. Rev. C 66, 034313 (2002).

[46] J. Schirmer, L. S. Cederbaum, and O. Walter, Phys. Rev. A 28 , 1237 (1983).

[47] A. Idini, F. Barranco, and E. Vigezzi, Phys. Rev. C 85, 014331 (2012).

[48] V. Somà, C. Barbieri, and T. Duguet, Phys. Rev. C 89, 024323 (2014).

[49] W. von Niessen, J. Schirmer, and L. Cederbaum, Comput. Phys. Rep. 1, 57 (1984).

[50] C. Barbieri, T. Duguet, and V. Somà (unpublished).

[51] D. R. Entem and R. Machleidt, Phys. Rev. C 68, 041001 (2003).

[52] R. Machleidt and D. R. Entem, Phys. Rep. 503, 1 (2011).

[53] P. Navrátil, Few-Body Syst. 41, 117 (2007).

[54] J. Fujita and H. Miyazawa, Prog. Theor. Phys. 17, 360 (1957).
[55] T. Duguet, H. Hergert, J. Holt, and V. Somà, arXiv:1411.1237[nucl-th] [Phys. Rev. C (to be published)].

[56] G. Audi, A. Wapstra, and C. Thibault, Nucl. Phys. A 729, 337 (2003).

[57] B. Jurado et al., Phys. Lett. B 649, 43 (2007).

[58] L. Gaudefroy et al., Phys. Rev. Lett. 109, 202503 (2012).

[59] Z. Vajta et al., Phys. Rev. C 89, 054323 (2014).

[60] C. R. Hoffman et al., Phys. Rev. Lett. 100, 152502 (2008).

[61] R. Kanungo et al., Phys. Rev. Lett. 102, 152501 (2009).

[62] Z. Elekes et al., Phys. Rev. Lett. 98, 102502 (2007).

[63] C. Caesar et al. (R3B Collaboration), Phys. Rev. C 88, 034313 (2013).

[64] A. Schiller et al., Phys. Rev. Lett. 99, 112501 (2007).

[65] M. Stanoiu et al., Phys. Rev. C 69, 034312 (2004).

[66] C. Barbieri, Phys. Lett. B 643, 268 (2006).

[67] S. C. Pieper and V. R. Pandharipande, Phys. Rev. Lett. 70, 2541 (1993).

[68] A. Gade et al., Phys. Rev. C 77, 044306 (2008).

[69] J. Lee et al., Phys. Rev. Lett. 104, 112701 (2010).

[70] F. Flavigny et al., Phys. Rev. Lett. 110, 122503 (2013).

[71] O. Jensen, G. Hagen, M. Hjorth-Jensen, B. A. Brown, and A. Gade, Phys. Rev. Lett. 107, 032501 (2011).

[72] E. Lunderberg et al., Phys. Rev. Lett. 108, 142503 (2012).

[73] C. Barbieri, A. Cipollone, V. Somà, T. Duguet, and P. Navrátil, arXiv:1211.3315.

[74] E. Caurier, K. Langanke, G. Martínez-Pinedo, F. Nowacki, and P. Vogel, Phys. Lett. B 522, 240 (2001).

[75] M. Avgoulea et al., J. Phys. G: Nucl. Part. Phys. 38, 025104 (2011).

[76] F. Barranco and R. a. Broglia, Phys. Lett. B 151, 90 (1985).

[77] A. Ekström, G. R. Jansen, K. A. Wendt, G. Hagen, T. Papenbrock, B. D. Carlsson, C. Forssén, M. Hjorth-Jensen, P. Navrátil, and W. Nazarewicz, Phys. Rev. C 91, 051301 (2015).

[78] A. Carbone, A. Polls, and A. Rios, Phys. Rev. C 88, 044302 (2013).

[79] A. Carbone, A. Rios, and A. Polls, Phys. Rev. C 90, 054322 (2014).

[80] A. L. Fetter and J. D. Walecka, Quantum Theory of ManyParticle System, 1st ed. (McGraw-Hill, New York, 1971).

[81] W. H. Dickhoff and D. Van Neck, Many-body Theory Exposed!, 2nd ed. (World Scientific, London, 2008).

[82] S. J. Waldecker, C. Barbieri, and W. H. Dickhoff, Phys. Rev. C 84, 034616 (2011).

[83] C. Barbieri and B. K. Jennings, Phys. Rev. C 72, 014613 (2005) 